\title{
2D ANGULAR CORRELATION OF ANNIHILATION RADIATION: CURRENT TRENDS AND FUTURE POSSIBILITIES
}

\author{
R.N. WEST
}

Department of Physics, University of Texas at Arlington, Arlington, TX 76019, USA

\begin{abstract}
Recent improvements in two-dimensional angular correlation measurement and analysis techniques have greatly increased the power of the method to image the Fermi surfaces of electronically complex systems. That, and the further and dramatic improvement that must result when the present radioisotope positron sources are replaced by intense monoenergetic positron beams are discussed and illustrated.
\end{abstract}

PACS numbers: 78.70.Bj, 71.28.+d, 71.25.Hc

\section{Introduction}

Over the past five to six years the extraordinary demands of cuprate high temperature superconductor measurements have stimulated improvements in two-dimensional angular correlation of annihilation radiation (2D ACAR) techniques that, in more normal circumstances, might well have taken two decades. In this paper we will briefly review those developments and how they have improved our capacity to image Fermi surfaces (FS) and other facets of electronic structure. We shall then show how our current dependence on positron radioisotope sources is limiting further development of the field. Finally, we shall show how the use, in ACAR, of high flux monoenergetic positron beams is going to further enhance the power of the technique.

\section{2D ACAR and its spectra}

In most contemporary 2D ACAR measurements, $\beta$-ray positrons from an external ${ }^{22} \mathrm{Na}$ source of $10-50 \mathrm{mCi}$ activity are constrained by their helical motion in an applied magnetic field to enter an oriented single crystal sample held in an appropriately constructed vacuum chamber. On entering the sample each positron rapidly thermalizes, and eventually annihilates via the two photon process, with one of the electrons of the system. The angular correlation of the emerging annihilation photon pairs is determined with a pair of opposed and distant (normally 10-20 m) position-sensitive detectors [1, 2] or detector arrays [3] working in a coincidence mode. The recorded histogram spectra represent $2 \mathrm{D}$ projections or integrals (along the major detector-sample-detector axis) of a 3D electron-positron momentum density $\rho_{\mathrm{ep}}(p)$ [3]. Because the positrons are thermal and repelled from the ion cores, the momenta involved are essentially those of the outer electrons 
and are $\approx 1-2 \mathrm{au}$. The momentum resolution, which is determined by the size of the sample, the position resolution of the detectors and the length of the photon flight paths, is usually better than 0.1 au. In most experiments the samples are oriented such that the integration direction is a high symmetry direction of the crystal.

$\rho_{\mathrm{ep}}(\boldsymbol{p})$ is closely related to the momentum density, $\rho_{\mathrm{e}}(\boldsymbol{p})$, of the electrons of the system. $\rho_{\mathrm{e}}(\boldsymbol{p})$ for a periodic solid is most easily described, in the single particle approximation, as a superposition of individual contributions from occupied states, of band index $n$ and reduced Bloch wave vector $k$. Those contributions occur at various points $\boldsymbol{p}=\hbar(\boldsymbol{k}+\boldsymbol{G})-\boldsymbol{G}$ is any reciprocal lattice vector - throughout the density, with relative intensities that are determined by the positron and electron wave functions. The consequent overall contribution from each band has the full point symmetry of the underlying crystal lattice and an overall shape that reflects the nature ( $s, p$ or $d$; core or conduction etc.) of the band in question. For a full band it is continuous. For a part-full band there are, in addition, discontinuities at all points $p_{\mathrm{F}}=\hbar\left(k_{\mathrm{F}}+G\right)$. The discontinuities mark the FS.

The degree to which one can resolve the FS or other significant structures in a measured 2D spectrum depends, of course, on the momentum resolution of the spectrometer, the statistical precision of the data and the nature of the material under study. In relatively simple metals with broad $s-p$ type conduction bands and distinct deep lying core bands, an image of the FS, of sufficient clarity for quantitative analysis, can usually be obtained in a $2 \mathrm{D}$ spectrum containing $\approx 5 \times 10^{7}$ counts. In the more complex transition or rare earth metals, the (once) integrated FS structures are less easily distinguished from anisotropies in both the full and part-full band contributions which arise from what we may loosely describe as single and many-particle "wave function" effects. In such cases two somewhat different reconstruction techniques have proved useful.

Tomography, the mathematical reconstruction of a $3 \mathrm{D}$ density distribution from its projections, is a well established technique in many fields of science. Its application to ACAR spectra is discussed extensively in Ref. [3]. Its virtues, in respect to the visualization of FS or other aspects of an electronic structure, are readily apparent in many of the existing results. The inevitable penalties (propagation of noise, degradation of resolution, etc.) which result from reconstruction are more than made up for by the benefits which result from the removal of the detail-obscuring integration of the FS structures. The only real deterrent, at the present time, is the considerable time required to produce the necessary multiple $2 \mathrm{D}$ spectra.

An alternative reconstruction approach of a rather different nature and based on a single spectrum, is the so-called Lock-Crisp-West (LCW) transformation [4]. In this transformation the $2 \mathrm{D}$ momentum density (or a reconstructed $3 \mathrm{D}$ density) is folded back into a primitive cell of the corresponding reciprocal lattice. The result is an equivalent reduced Bloch wave vector, $k$-space density. In this $k$-space density, the FS structures which were originally distributed throughout the original momentum density at the various $p_{\mathrm{F}}=\hbar\left(\boldsymbol{k}_{\mathrm{F}}+\boldsymbol{G}\right)$ are brought together to form a set of coherent and reinforced images of the FS in the first Brillouin zone. The continuous parts of the momentum density sum into a comparatively flat and 
featureless background. That said, there are, in that background, residual wave function effects which can sometimes obscure and/or distort the FS images. We cannot model those distortions exactly because of the many-body effects but we can argue from symmetry and other general considerations that the functional form is a superposition of a few long wavelength cosines. That is now clear in numerous results and enables us (see Sec. 3.2) to suppress them almost entirely.

\section{Contemporary problems and solutions}

\subsection{Measurements}

Over the period 1975 to 1988 a wide range of studies of elemental metals and binary disordered substitutional alloys clearly established the ability of the 2D ACAR technique, sometimes complemented by $3 \mathrm{D}$ reconstruction or, more frequently, by LCW folding, to image an entire FS and, in some cases, provide additional information about the nature of the associated electron energy bands. More recently it has been applied to compounds and problems of much greater complexity. The ceramic high-temperature superconductors are a case in point. These materials satisfy few of the established criteria for a good ACAR measurement. The specimens are frequently so small (typically $1 \mathrm{~mm}^{2}$ in area) and so thin $(\approx 50 \mu \mathrm{m})$ as to make it difficult to stop the positrons in them rather than in the supporting substrate. Their multi-element bases, consequent large unit cells and small Brillouin zones, and multiplicity of bands create the need for exceptionally high momentum sampling density and statistical precision in every spectrum measurement. Even with that, the measured spectra invariably turn out to be, at least as compared to those for elemental metals, almost isotropic and structure free. Were that not enough, the crystals invariably contain open volume defects in which there is the possibility of positron trapping to complicate the interpretation of results.

Partial answers to most of these difficulties have been found, albeit at some cost. Imaginative solutions to the very small sample problem involving such devices as large "pseudo-crystal" arrays of several smaller single crystals and/or sample mesh "substrates" made from ultra-fine (10-20 mm diameter) wires [5] have been devised. Computer hardware and software improvements have permitted a progressive increase in the size of the data arrays (from the original $4 \mathrm{~K}$ to $16 \mathrm{~K}$ and, occasionally, to $64 \mathrm{~K}$ addresses) and a corresponding decrease in the bin widths (now down to the order of $10^{-2}$ au). Concomitantly, the statistical precision has been increased by a factor of 3 or 4 by extending the data accumulations up to $\approx 10^{9}$ coincidence counts. Those various improvements in the technique were forced by the exceptional needs of the superconductor research but they are now being applied more widely. To complement that we find similar advances in data processing and analysis.

\subsection{Analyses}

For many years the identification of FS structures in 2D ACAR results was largely based on visual examinations of $1 \mathrm{D}$ sections, isometric images and 2D contour diagrams of the spectra. That approach worked reasonably well for the 
elemental metals and binary alloys but has become increasingly inadequate for, as the materials of interest have become chemically more complex, the FS structures have become progressively smaller both in absolute intensity and in comparison to the residual wave function effects. However, at the same time, comprehensive software packages that enable rapid processing and imaging of large data arrays have become generally a vailable. They, together with the much improved statistical precision of contemporary data have greatly enhanced our ability to separate the small FS discontinuities from the remaining wave function signals in the presence of both instrumental and other spectrum smearing effects and the residual noise. The ACAR groups at the Universities of Geneva and Bristol have both developed sophisticated procedures $[6,7]$ for the deconvolution of instrumental smearing effects from spectra using a combination of maximum entropy and least squares criteria. Such deconvolution enhances the FS images and, in theoretically well understood systems, usually results in an ACAR spectrum almost indistinguishable from that predicted by a self-consistent bandstructure theory (Fig. 1). The Geneva group are also using a variety of other linear and non-linear filter

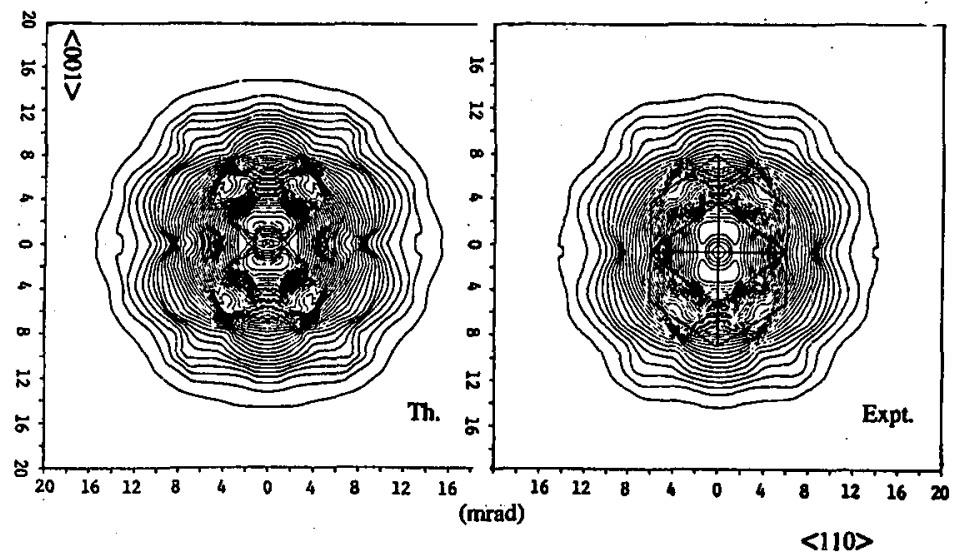

Fig. 1. Contour diagrams of deconvoluted (see text) experimental [7] and theoretical [8] 2D momentum densities for vanadium. The resolved momentum components are indicated.

methods for noise smoothing and data modeling [7]. In our laboratory the major preoccupation has been the separation of the FS images from the residual wave function cosine (see Sec. 2) signals in the LCW spectra.

In a series of studies of real and simulated spectra we have examined the relative merits of a variety of different procedures aimed at the enhancement of the FS images, with an emphasis upon those which require little or no prior knowledge about the specific form of those images. The most efficacious has proved to be a simple and rapidly applicable frequency limited derivative (FLD) technique [9] which suppresses the high frequency noise and the essentially low frequency (long wavelength) error signals leaving a clear and almost distortion-free image of the FS discontinuities. We developed it for and have found it effective in studies of su- 
perconductors [10] and other compounds [11] but its benefits are also clear (Fig. 2) in metals and alloy spectra where the FS signals are initially obscured by large cosine signals. The implications are clear. The single and many particle effects need no longer compromise a Fermi surface interpretation of LCW spectra.

(a)

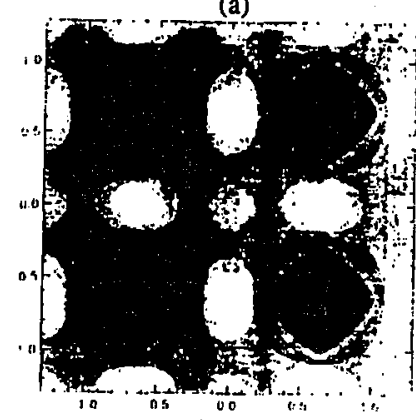

(a. u.) (b)

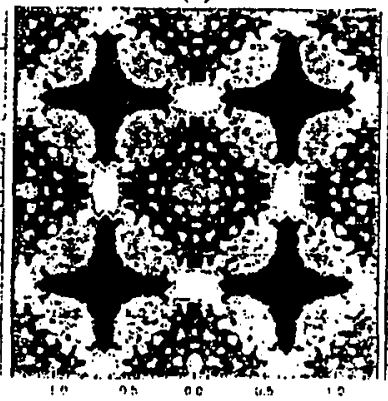

(a. u.)

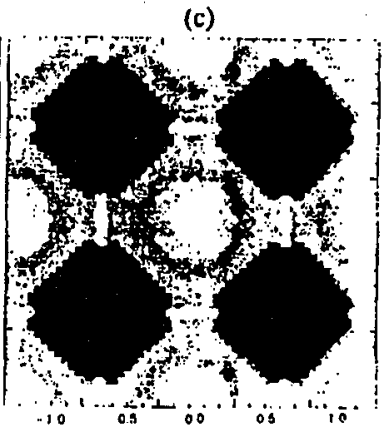

(a. u.)

Fig. 2. LCW spectra for Cu, [100] integration. (a) Symmetry folded experimental data. (b) FLD filtered (see text) data. (c) Theoretical spectrum according to Brand (unpublished).

\section{ACAR and positron beams}

The imaging power of the 2D ACAR technique has increased considerably in the past few years and developments in detector technology, that could further improve both the counting efficiency and resolution of ACAR machines are in the offing. But the full exploitation of these developments is now inhibited by the conventional radioisotope positron source. The supply of those sources is unreliable, their quality is variable, and, most significantly, their emission characteristics are quite inadequate for our developing needs. Major problems arise from our inability to confine the available positron flux to a cross-section less than that of the active area (typically $\approx 15 \mathrm{~mm}^{2}$ ) of the source. To maximize the number of positrons entering the specimen, and thus the data rate, the specimen area, $A_{\mathbf{s}}\left(\approx d_{\mathrm{s}}^{2}\right)$, needs to be greater than that of the source $A_{\mathrm{f}}\left(\approx d_{\mathrm{f}}^{2}\right)$. But the linear dimensions of the specimen, as seen by the detector, also affect the overall angular resolution [12]. If the detector position resolution FWHM is $d_{d}$, an easily argued criterion for the optimal efficiency and resolution is $d_{\mathrm{f}}<d_{\mathrm{s}}<d_{\mathrm{d}}$. That simple condition was achievable $\left(d_{\mathrm{f}} \approx 4 \mathrm{~mm}, d_{\mathrm{d}} \approx 5 \mathrm{~mm}\right)$ for a long time, but, today, with progressively smaller (1 mm or less) samples demanding attention and steadily improving detector resolutions $\left(d_{\mathrm{d}} \approx 2-3 \mathrm{~mm}\right)$, it is impossible to satisfy for there seems no way that the manufacturers of the sources can make them any smaller. If we keep $d_{s}$ large we degrade the overall resolution. If we choose, or are compelled (the more likely scenario), to make it small, we suffer a profound loss of counting efficiency. The continuous energy spectrum of the $\beta$-ray positrons and their consequent deep penetration depths is another major limiting factor when so many of the most important materials science problems involve thin films or surfaces. Nevertheless there is an alternative to the radioisotope source. 
Another important contemporary sub-field of positron physics is concerned with the creation and exploitation of mono-energetic positron beams [13]. A small number of ACAR surface studies [14-16] using medium flux $\left(10^{7}-10^{8} \mathrm{e}^{+} \mathrm{s}^{-1}\right)$ beams of low energy positrons have shown the possibility for a whole new class of powerful positron and positronium spectroscopies. At present those new spectroscopies are made difficult by the lack of sufficient positron flux. But, the technology now exists whereby one can realistically contemplate ultra-high flux $\left(\approx 10^{10} \mathrm{e}^{+} \mathrm{s}^{-1}\right)$, small cross-section $\left(<0.1 \mathrm{~mm}^{2}\right)$ variable energy positron beams. With such beams we could both overcome the present constraints on tenable specimens for bulk physics studies and attack serious problems in surface and thin film physics.

The magnitude of our existing source problem and the benefits of a high flux beam can be readily quantified. Our ability to resolve in any measurement, for example, a 1D (edge) discontinuity in the presence of statistical noise can be expressed, in terms of the total number of counts in the spectrum $N$ and the overall resolution FWHM $\omega$, through an imaging factor $Q=N^{1 / 2} \omega^{-1}$ (for 2D structures $\omega^{-2}$ is more appropriate). $Q$ can be also defined, in terms of the primary positron flux $N_{0}$, the duration $t$ of the experiment, and a reduced spectrometer imaging factor $q$; as $Q=N_{0}^{1 / 2} \tau^{1 / 2} q . q$ is a dimensionless function of various geometrical factors including the various $d$ 's already defined above and the detector efficiencies. It can be readily computed for any machine [12].

TABLE

Relative imaging factors, $q$ (upper left corner) and accumulation times, $\tau$ (lower right corner) for a selection of different source $\left(d_{\mathfrak{f}}\right)$, sample $\left(d_{\mathrm{s}}\right)$ and detector resolution $\left(d_{\mathrm{d}}\right)$ parameters, relevant to the situations pertaining and/or likely to pertain to ACAR systems in the years shown.

\begin{tabular}{|c|c|c|c|c|}
\hline \multirow{2}{*}{$\begin{array}{r}\text { Detector, } \\
d_{\mathbf{s}}[\mathrm{mm}]\end{array}$} & ${ }^{22} \mathrm{Na}$ & $d_{\mathrm{f}}=4 \mathrm{~mm}$ & Beam & $d_{\mathrm{f}}=0.5 \mathrm{~mm}$ \\
\hline & $d_{\mathrm{s}}=3 \mathrm{~mm}$ & $d_{\mathrm{s}}=1 \mathrm{~mm}$ & $d_{\mathrm{s}}=3 \mathrm{~mm}$ & $d_{\mathrm{s}}=1 \mathrm{~mm}$ \\
\hline \multirow{2}{*}{$1982,6.5$} & 0.21 & 0.08 & 0.30 & 0.30 \\
\hline & $25 \mathrm{~d}$ & $5.8 \mathrm{~m}$ & $8.8 \mathrm{~h}$ & $8.8 \mathrm{~h}$ \\
\hline \multirow{2}{*}{$1992,3.4$} & 0.33 & 0.14 & 0.56 & 0.56 \\
\hline & $10 \mathrm{~d}$ & $56 \mathrm{~d}$ & $2.5 \mathrm{~h}$ & $2.5 \mathrm{~h}$ \\
\hline \multirow{2}{*}{$2002,2.0$} & 0.42 & 0.22 & 0.89 & 0.89 \\
\hline & $6 \mathrm{~d}$ & $23 \mathrm{~d}$ & $1.0 \mathrm{~h}$ & $1.0 \mathrm{~h}$ \\
\hline
\end{tabular}

In Table we have listed the relative $q$ 's for the machines we operated in 1982 and 1992 and that we expect to have available in or before 2002, and for 
relevant values of $d_{\mathrm{f}}, d_{\mathrm{s}}$ and $d_{\mathrm{d}}$. We have computed them both for a radioisotope source with $d_{\mathrm{f}} \approx 4 \mathrm{~mm}$ and for a beam with $d_{\mathrm{f}} \approx 0.5 \mathrm{~mm}$. We have assumed no other improvements in the position-sensitive detector technology other than in their resolution. The implications are clear. If we continue to use the radioisotope sources, the benefits of 20 years of progressively improving detector resolution are going to be completely negated by the inevitable trend towards smaller and smaller specimens. With, a beam, on the other hand, they can be exploited to the full. The benefits are even more apparent when we consider the matter in terms of data accumulation times.

We have also included in Table a further indication of the full potential of a beam based ACAR system in the form of the relative data accumulation times required to produce the same quality factor $Q$, with a typical ACAR radioisotope source $\left(N_{0}=3 \times 10^{8} \mathrm{e}^{+} \mathrm{s}^{-1}\right)$ and with a beam with $N_{0}=10^{10} \mathrm{e}^{+} \mathrm{s}^{-1}$. Such a beam is long overdue. It will involve appreciable capital expenditure and, given that, a more ambitious ACAR measurement system is probably justified. Today, we can build an ACAR machine for about one tenth of the cost (in real terms) that obtained in 1976. Ten machines, set up at regular angles spanning a point symmetry element of the specimen crystal would provide the data for a real-time, on-line reconstruction of the entire 3D momentum density. Conservatively, we may assume the quality factor of such a measurement continues to scale as the square root of the overall counts. With that combination of high intensity positron beam and multi-projection ACAR machine we would achieve, as compared with our capability today, a magnification in $Q$ of the order of 50 . Equivalently, the benefits of a 30 day study on our current machine (Table) could be obtained in just a few mirutes.

\section{Conclusions}

During the past few years the imaging power of the positron 2D ACAR technique has been much improved by developments in both the measurement techniques and in the data processing, analysis and visualization. The only serious obstacle to the further development of the technique is the conventional radioisotope source. The combination of a high flux positron beam and a super ACAR system would provide an electronic structure imaging system of unparalleled power and rapidity.

\section{Acknowledgments}

The author would like to thank Mr. Kevin O'Brien for permission to use his results prior to their full publication and for help in preparing the figures.

\section{References}

[1] R.N. West, J. Mayers, P.A. Walters, J. Phys. E, Sci. Instrum. 14, 478 (1981).

[2] A.A. Manuel, S. Samoilov, O. Fischer, M. Peter, A.P. Jeavons, Helv. Phys. Acta 55, 100 (1979).

[3] S. Berko, in: Positron Solid State Physics, Eds. W. Brandt, A. Dupasquier, North-Holland, Amsterdam 1983, p. 64. 
[4] D.G. Lock, V.H.C. Crisp, R.N. West, J. Phys. F 3, 561 (1975).

[5] H. Haghighi, J.H. Kaiser, S. Rayner, R.N. West, J.Z. Liu, R. Shelton, R.H. Howell, F. Solal, M.J. Fluss, Phys. Rev. Lett. 67, 382 (1991).

[6] L. Hoffmann, A. Shukla, M. Peter, B. Barbiellini, A.A. Manuel, Nucl. Instrum. Methods Phys. Res. A, in press.

[7] S.B. Dugdale, M.A. Alam, H.M. Fretwell, M. Biasini, D. Wilson, J. Phys., Condens. Matter 6, L435 (1994).

[8] M. Matsumoto, S. Wakoh, J. Phys. Soc. Jpn. 55, 3948 (1986).

[9] K.M. O'Brien, M.Z. Brand, S. Rayner, R.N. West, J. Phys., Condens. Matter 7, 1 (1995).

'10] R.N. West, in: Metallic Alloys: Experimental and Theoretical Perspectives, Eds. J.S. Faulkner, R.G. Jordan, Kluwer, Dordrecht 1994, p. 65.

[11] M. Biasini, M.A. Alam, H. Harima, Y. Onuki, H. Fretwell, R.N. West, J. Phys., Condens. Malter 6, 7823 (1994).

[12] R.N. West, in: Positron Spectroscopy of Solids, Eds. A. Dupasquier, A.P. Mills Jr., North-Holland, Amsterdam 1995, in press.

[13] P.J. Schultz, K.G. Lynn, Rev. Mod. Phys. 60, 701 (1988).

[14] R.H. Howell, P. Meyer, I.J. Rosenberg, M.J. Fluss, Phys. Rev. Lett. 54, 1698 (1985).

[15] K.G. Lynn, A.P. Mills, R.N. West, S. Berko, K.F. Canter, L.O. Roellig, Phys. Rev. Lett. 54, 1702 (1985).

[16] D.M. Chen, S. Berko, K.F. Canter, K.G. Lynn, A.P. Mills, L.O. Roellig, P. Sferlazzo, M. Weinert, R.N. West, Phys. Rev. Lett. 58, 921 (1987). 